Methods OSHI accepts referrals from community and secondary care and is targeted to those with moderate-severe alcohol dependence and recent hospital attendance. A review of the first 9 months was undertaken in June 2021. Demographic details were collated, alongside data including numbers engaging, and/or achieving abstinence. We also sought user feedback via a structured survey.

Results 48 patients were referred to OSHI over 9 months [31\% female; mean age 45 (24-60)]. Across all referrals, 23 $(47.9 \%)$ were currently abstinent with the majority $(n=21$; 91.3\%) being OSHI users. 31 patients (64.6\%) were actively engaging with OSHI. Of those, 21 (67.7\%) were abstinent which compares favourably to rates achieved through CASS. More than half of abstainers $(n=12 ; 57.1 \%)$ engaged with OSHI exclusively. For the $14(29.2 \%)$ patients using OSHI not previously known to CASS, engagement and abstinence were particularly high $(\mathrm{n}=12 ; 85.7 \% ; \mathrm{n}=9 ; 75 \%$, respectively). Patient feedback was uniformly excellent. Everyone welcomed the personalized approach. Many highlighted the on-demand support ('you're never alone...'; 'a friend...rather than....an official appointment'; '...can call, even just to chat'), as well as the perceived informality compared to other services. Conclusions The results show high rates of engagement and abstinence with OSHI. We will next determine if rates are maintained longer term, and work to better understand reasons for non-engagement with CASS/OSHI, so interventions are targeted effectively. We hope to expand OSHI to include mentors from a variety of backgrounds and life experiences, and to enable referrals from other sources. Our team has been enriched by the integration of a peer-support volunteer who brought fresh perspective and ideas and in doing so, helped many others to control their drinking. We wholeheartedly recommend this approach to other services.

\section{P026 DIFFERENTIAL EPIGENETIC REGULATION IN SURVIVORS AND NON-SURVIVORS FROM SEVERE ALCOHOLIC HEPATITIS}

${ }^{1}$ Huey Tan*, ${ }^{2}$ Matthew Cramp, ${ }^{2}$ Ashwin Dhanda. ${ }^{1}$ Hepatology Research Group, University of Plymouth, Plymouth, UK; ${ }^{2}$ South West Liver Unit, Plymouth University NHS Trust, Plymouth, UK

10.1136/gutjnl-2021-BASL.35
Background and Aims Alcohol is known to indirectly influence immune function through epigenetic regulation, but if this has an impact on alcoholic hepatitis (AH) remains unknown. The Histone deacetylase (HDACs) and histone acetylase (HATs) gene families differentially regulate histone accessibility to key pro- and anti-inflammatory transcription factors, a mechanism for epigenetic control. We hypothesised that differential expression of HDAC and HAT genes may influence susceptibility to and outcome from $\mathrm{AH}$.

Method Patients from University Hospitals Plymouth with $\mathrm{AH}$ (new jaundice, coagulopathy, heavy alcohol use, discriminant function $[\mathrm{DF}]>32$ ) and healthy volunteers (HV) were recruited. Model for end stage liver disease (MELD) and DF scores were used to evaluate liver disease severity. Disease outcome was determined with 90-day mortality. Peripheral blood, obtained at baseline, was separated by magnetic beads into CD4+ $\mathrm{T}$ cells and CD14+ monocytes. HDAC (HDAC1-11, SIRT 1-7) and HAT (GCN5, PCAF, p300, CBP, SRC-1) gene expression was assessed by qPCR in each immune subset. Expression was normalised to GAPDH and expressed relative to pooled cDNA from 5 healthy volunteers (HVs).

Results 15 AH patients were recruited (10 males; mean age 53.8; mean DF 80.6), of which 5 were dead within 90 days. HV cDNA were pooled from 5 individuals $(3$ males; Mean age 36).

When comparing $\mathrm{AH}$ survivors to non-survivors in CD14+ monocytes, HDAC 2, 3, 8

SIRT $1,2,5,6,7$ were significantly upregulated in the survivor group.

In CD4+ T cells, HDAC 2, 8 expression levels were significantly higher in the survivor group, while HDAC 11 was significantly higher in the non-survivor group (figure 1).

When investigating the HAT, GCN5 and SRC-1 were significantly upregulated in $\mathrm{CD} 14+$ monocytes in $\mathrm{AH}$ survivors compared to non-survivors (figure 1).

In $\mathrm{CD} 4+\mathrm{T}$ cells, $\mathrm{AH}$ non survivors have significantly upregulated GCN5, p300, CBP and SRC-1, levels when compared to $\mathrm{AH}$ survivors (figure 1 ).

The rest of the genes listed are not differentially expressed in both cohort of $\mathrm{AH}$ survivors and non-survivors.

Conclusion These novel data demonstrate that HDAC and HAT genes are differentially expressed in AH survivors compared to non-survivors. Histone acetylation and deacetylation may play a mechanistic role in driving disease pathogenesis or
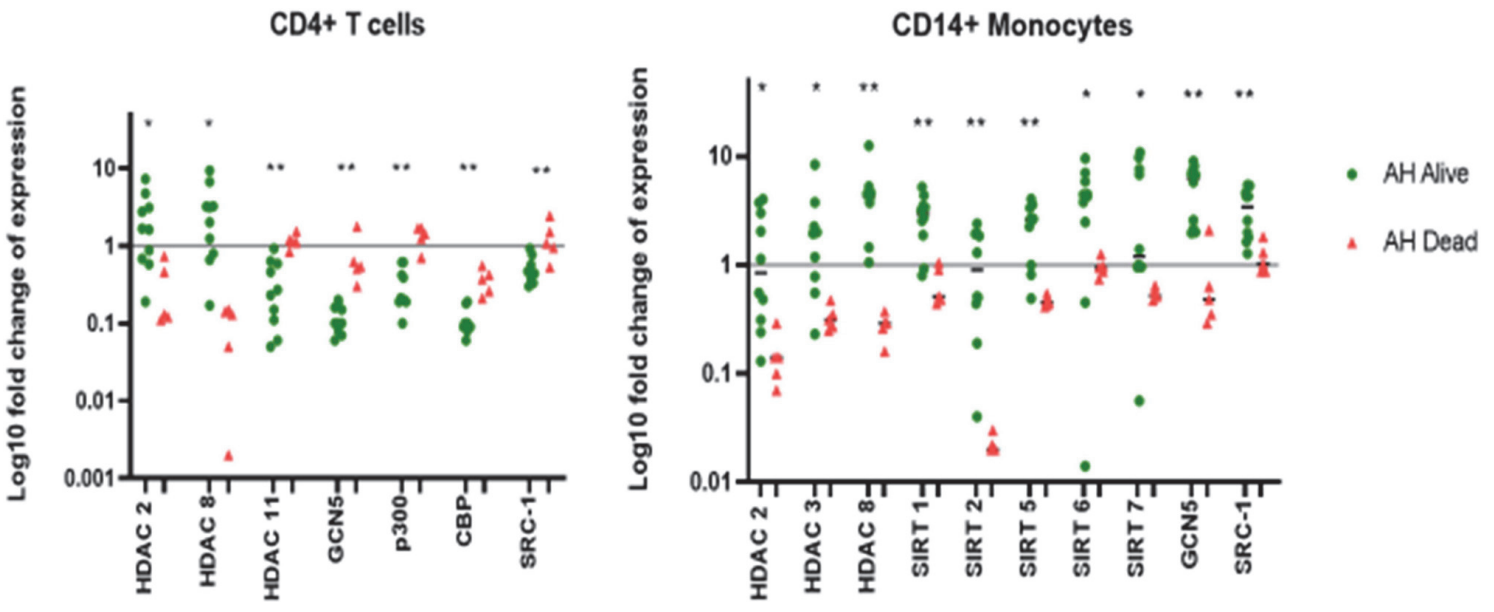

Abstract P026 Figure $1{ }^{*}-p<0.05,{ }^{* *}-p<0.01$ 
resolution. Further studies to investigate these changes may help reveal potential therapeutic targets for $\mathrm{AH}$.

\section{P027 RETROSPECTIVE ANALYSIS OF BONE PROTECTION CARE IN PRIMARY BILIARY CHOLANGITIS (PBC) IN A DISTRICT GENERAL HOSPITAL}

Yun Jie Chew*, John Hutchinson. Mid York NHS Trust, UK

\subsection{6/gutjnl-2021-BASL.36}

Introduction We performed a review of bone management and follow up among patients with Primary Biliary Cholangitis (PBC) at Mid Yorkshire Hospitals NHS Trust (MYHT). MYHT provides hospital services to a population of 530,000. Methods We undertook a retrospective analysis of 542 patient records and clinic letters through Cito, which is the Trust's electronic Document Management System and ICE system. We included patients who were under the outpatient care of both the Hepatology and Gastroenterology services over a 3 year period from December 2017 to December 2020.

Results In term of bone care, 54\% $(n=21)$ had up to date bone density scan (DEXA), 33\% $(n=13)$ have never had a DEXA scan and 13\% $(n=5)$ have had DEXA scan in the past but were due for a repeat scan. Among those who had a DEXA scan ( $n=26$ and 67\%), 42\% $(n=11)$ of them had osteoporosis and 31\% $(n=8)$ had osteopenia.

In respect to $\mathrm{PBC}$ patients diagnosed with osteoporosis or osteopenia, only $10 \%(n=2)$ were not on any bone protection therapy. This includes bisphosphonates, calcium and vitamin D replacement. However, depending on the serum level of calcium and vitamin D of patient, they are able to get calcium and vitamin D over the counter and therefore not prescribed by hospital or GP. Calcium and Vitamin D replacement is essential since there is sufficient evidence suggesting calcium and vitamin D alone prevent bone loss and reduce fracture. ${ }^{1}$ Discussions It was recommended by National Institute for Health and Care Excellence (NICE) that after a minimum of 2 years, risk assessment need to be undertaken among the population whose original calculated FRAX score was in the region of the intervention threshold for treatment. It was unsure whether they have had a risk assessment for osteoporosis since the Fracture Risk Assessment Tool (FRAX) score was not documented in clinic letters. ${ }^{2}$ Therefore, we need to ensure risk assessment for osteoporosis is performed in the clinic and documented clearly in the clinic letter using the FRAX score.

\section{REFERENCES}

1. Sunyecz JA. The use of calcium and vitamin $D$ in the management of osteoporosis. Ther Clin Risk Manag 2008;4(4):827-836. doi:10.2147/tcrm.s3552

2. National Institute of Health and Care Excellence (NICE). Osteoporosis: assessing the risk of fragility fracture [CG146] 2017. Available from: https://www.nice.org. uk/guidance/cg146 [Accessed 28th May 2021]

\begin{tabular}{ll} 
Abstract P027 Table 1 & \\
\hline & Number of patients $(\mathrm{n}=39)$ \\
\hline Bone protection & 12 \\
Osteoporosis & 8 \\
Osteopenia & 7 \\
Normal & \\
\hline
\end{tabular}

\section{P028 CHRONIC LIVER DISEASE, PLATELET TRANSFUSION \& QUALITY OF LIFE - A BRITISH LIVER TRUST PATIENT- SURVEY}

'Jonathan Worsfold, 'Vanessa Hebditch, ${ }^{2}$ Stephen Ryder. 'British Liver Trust, UK; ${ }^{2}$ Queen's Medical Centre, Nottingham, UK

\subsection{6/gutjnl-2021-BASL.37}

Introduction For patients with chronic liver disease, thrombocytopenia is a common complication that impacts on management of cirrhosis and has implications for planned surgical/ diagnostic procedures due to an increased risk of bleeding. Platelet transfusion (PT) has been the standard of care for management of thrombocytopenia. There is a need to better understand the views and experiences of UK patients with advanced disease and how their condition, and requirements for PT, affect quality of life.

Method The British Liver Trust (BLT) undertook a patient survey between December 2020 and February 2021. Patients were invited to participate via BLT channels (monthly newsletter; patient support groups) answering 30 questions (anonymously) via the Survey Monkey platform. The survey was conducted independently by the BLT, supported by an educational grant from Shionogi.

Results A total of 121 patients completed the survey. Alcohol related liver disease was the most commonly reported primary liver disease $(28 \%) ; 63 \%$ of respondents were female; $73 \%$ were aged over 45 years, and $51 \%$ have been told they may require a liver transplant in the future. A large proportion $(69 \%)$ of patients reported that liver disease sometimes affects their ability to think clearly. Half of responders recorded having to take time off work for liver-related hospital appointments; $40 \%$ travel $>25$ miles to their liver centre/hospital for routine appointments, and 55\% said that the COVID-19 pandemic had led to procedures related to their liver disease being cancelled. Many patients (62\%) reported that healthcare professionals (HCPs) have difficulties inserting a needle into a vein and $40 \%$ noted that they have needed a dental extraction since being diagnosed. Almost a third (30\%) have required a PT at some time, and of those, $70 \%$ had to stay in hospital the night before the PT. Patients reported that the reasons for needing a PT were typically well-explained. Among recipients of $\mathrm{PT}, 42 \%$ said they felt unwell during or post the PT. Following completion of the survey, 33 patients provided consent for voluntary follow-up and supplementary questions from the BLT.

Conclusions A survey of patients with chronic liver disease highlights that the COVID-19 pandemic has impacted on and delayed planned procedures, and identifies that both chronic liver disease, and the requirement for PT, pose a burden to patients that may adversely affect quality of life.

\section{P029 PLASMALEMMA VESICLE-ASSOCIATED PROTEIN (PLVAP) MEDIATES MONOCYTE TRANSMIGRATION ACROSS HUMAN HEPATIC SINUSOIDAL ENDOTHELIUM IN RESPONSE TO THE SENESCENT SECRETOME}

${ }^{1}$ Alex Wilkinson*, ${ }^{1}$ Daniel Patten, ${ }^{1}$ Sam Hulme, ${ }^{2}$ Matthew Hoare, ${ }^{1}$ Shishir Shetty. ${ }^{1}$ University of Birmingham, Institute of Immunology and Immunotherapy, UK; ${ }^{2}$ University of Cambridge, Department of Medicine, UK

10.1136/gutjnl-2021-BASL.38 